\title{
Site-Directed Mutagenesis at the Molybdenum Pterin Cofactor Site of the Human Aldehyde Oxidase: Interrogating the Kinetic Differences Between Human and Cynomolgus Monkey ${ }^{\S}$
}

\author{
Armina Abbasi, Carolyn A. Joswig-Jones, and Jeffrey P. Jones \\ Department of Chemistry, Washington State University, Pullman, Washington
}

Received July 17, 2020; accepted September 25, 2020

\section{ABSTRACT}

The estimation of the drug clearance by aldehyde oxidase (AO) has been complicated because of this enzyme's atypical kinetics and species and substrate specificity. Since human $A O$ (hAO) and cynomolgus monkey $A O$ ( $\mathrm{MAO}$ ) have a $95.1 \%$ sequence identity, cynomolgus monkeys may be the best species for estimating $A O$ clearance in humans. Here, $0^{6}$-benzylguanine (O6BG) and dantrolene were used under anaerobic conditions, as oxidative and reductive substrates of $A O$, respectively, to compare and contrast the kinetics of these two species through numerical modeling. Whereas dantrolene reduction followed the same linear kinetics in both species, the oxidation rate of O6BG was also linear in $\mathrm{MAO}$ and did not follow the already established biphasic kinetics of hAO. In an attempt to determine why hAO and $\mathrm{mAO}$ are kinetically distinct, we have altered the hAO V811 and F885 amino acids at the oxidation site adjacent to the molybdenum pterin cofactor to the corresponding alanine and leucine in $\mathrm{mAO}$, respectively. Although some shift to a more monkey-like kinetics was observed for the V811A mutant, five more mutations around the AO cofactors still need to be investigated for this purpose. In comparing the oxidative and reductive rates of metabolism under anaerobic conditions, we have come to the conclusion that despite having similar rates of reduction (4-fold difference), the oxidation rate in $\mathrm{mAO}$ is more than $\mathbf{5 0}$-fold slower than hAO. This finding implies that the presence of nonlinearity in AO kinetics is dependent upon the degree of imbalance between the rates of oxidation and reduction in this enzyme.

\section{SIGNIFICANCE STATEMENT}

Although they have as much as $95.1 \%$ sequence identity, human and cynomolgus monkey aldehyde oxidase are kinetically distinct. Therefore, monkeys may not be good estimators of drug clearance in humans.

\section{Introduction}

Aldehyde oxidase (AO) is a molybdoflavoprotein mostly found in three different oligomeric states as a $150 \mathrm{kDa}$ monomer, a homodimer, and a tetramer (Mendel, 2009; Mota et al., 2019). In recent years, efforts to make drugs less susceptible to metabolism by the cytochrome P450 family of enzymes, which act via electrophilic attack, have led to an increase in substrates with a higher susceptibility to AO metabolism, which occurs via nucleophilic attack (Dowers et al., 2004; Alfaro and Jones, 2008; Pryde et al., 2010; Davydova et al., 2019).

AO is capable of oxidation of a wide range of compounds such as aldehydes, alicyclic iminium ions, and aromatic azaheterocycles (Pryde et al., 2010), and the oxidation half-reaction is believed to occur close to the molybdenum pterin cofactor (MoCo) site (Coelho et al., 2015). Molecular oxygen is assumed to be the final electron acceptor in the AO catalytic cycle (Alfaro and Jones, 2008; Pryde et al., 2010). Until recently it was believed that reduction of other substrates, such as the ones containing nitro groups, by AO would only happen under anaerobic

This work was supported by National Institutes of Health National Institute of General Medical Sciences [GM100874].

https://doi.org/10.1124/dmd.120.000187.

S This article has supplemental material available at dmd.aspetjournals.org. conditions (Li et al., 2009; Weidert et al., 2014; Maia et al., 2015). However, quite a few examples of aerobic nitro reduction have been presented in the literature in the past few years (Konishi et al., 2017; Amano et al., 2018; Ogiso et al., 2018). This has in turn brought up the possibility of a competition between the reductive substrates and the molecular oxygen at the flavin adenine dinucleotide (FAD) site (Paragas et al., 2017a).

Many promising drug candidates are removed from the pipeline or are chemically modified due to poor estimation of their metabolism by AO in humans; examples include BIBX1382 (Dittrich et al., 2002), SGX523 (Diamond et al., 2010), and RO1 (Zhang et al., 2011). One commonly used preclinical method for estimation of drug clearance in humans is the use of interspecies allometric scaling (Nair and Jacob, 2016). However, this approach, which is based on scaling by weight, is limited as it ignores several factors such as protein binding, physicochemical properties of the drug, and interspecies differences in metabolism and transport (Sharma and McNeill, 2009). Despite efforts to incorporate these absorption, distribution, metabolism, and excretion parameters into a single animal model for estimation of AO clearance in humans, trends observed to date have not been generalizable. Consequently, alternative methods such as in vitro scaling or multiple species allometry have been suggested for this enzyme (Zientek et al., 2010; Crouch et al., 2018). The complicated interspecies differences for AO may have arisen

ABBREVIATIONS: ADNTN, aminodantrolene; AIC, Akaike information criterion; AO, aldehyde oxidase; AOX, aldehyde oxidase gene; FAD, flavin adenine dinucleotide; hAO, purified expressed human aldehyde oxidase; MAM, modified activity model; mAO, purified expressed cynomolgus monkey aldehyde oxidase 1; MoCo, molybdenum pterin cofactor; OAR, [8-oxo-benzylguanine] to [aminodantrolene] ratio; O6BG, ${ }^{6}$ benzylguanine; TDI, time-dependent inhibition. 
TABLE 1

The human to monkey (hAO\#mAO) mutants were made using the primers, DNA templates, and the sequencing primers as described

\begin{tabular}{lccc}
\hline Plasmid (hAO\#mAO) & Primer & DNA template & Sequencing primer \\
\hline F885L & FW: 5'-cagacccattcaataactaacaggcttcatccagg-3' & pTHco-AOX1 & $5^{\prime}$-attgttgccagcacactgaa-3' (nucleotides 2401-2800) \\
& RV: 5'-cctggatgaaagcctgttagttattgaaatgggtctg-3' & & \\
V811A & FW: 5'-aataataccggtttcagcgcttaccaccaaatgcacc-3' & pTHco-AOX1 & \\
& RV: 5'-ggtgcatttggtggtaagcgctgaaaaccggtattatt-3' & F885L & \\
FLVA & FW: 5'-aataataccgtttcagcgcttaccaccaaatgcacc-3' & & \\
& RV: 5'-ggtgcatttggtggtaaagcgctgaaaaccggtattatt-3' & & \\
\hline
\end{tabular}

due to the presence of various isoforms in different species or the multiple active sites present in this enzyme (Hoshino et al., 2007; Garattini et al., 2009; Paragas et al., 2017a).

The number of AOX active genes in traditional preclinical species varies from none in dog hepatocytes to the extreme of four in rodents (Garattini et al., 2009). When more than one AO isozyme is present in a species, they typically exhibit tissue specificity (Cheshmazar et al., 2019). In humans, only the AOX 1 gene shows activity, and the other two pseudogenes (AOX2 and AOX4) remain inactive despite being transcribed. The same trend is observed in chimpanzees, demonstrating that this functional inactivation of AOX 4 and AOX2 occurred before the human speciation occurred (Garattini et al., 2009; Cheshmazar et al., 2019). Depending on the substrate, various species exhibit different levels of activity. For example, rabbit metabolizes methotrexate and cinchonidine several orders of magnitude faster than rhesus monkey, and monkey is faster than human (Itoh et al., 2006b; Choughule et al., 2015). Alternatively, a rank order of rabbit, human, monkey was assigned to zoniporide (Dalvie et al., 2013). Furthermore, studies on several other substrates such as zonisamide, (S)-RS-8359, phthalazine, and N-(2-dimethylamino)ethyl) acridine-4-carboxamide (DACA) have observed the highest AO activity in monkeys and humans, followed by rabbits, guinea pigs, and rodents (Itoh et al., 2006a; Fukiya et al., 2010; Choughule et al., 2013). To learn more about AO species differences, we sought to understand whether specific amino acids at the oxidation active site in humans and nonhuman primates were contributing to the divergent activities observed. Cynomolgus monkeys share a $95.1 \%$ AO amino acid sequence identity with humans, and their small size makes this a species of high interest (Hoshino et al., 2007). Furthermore, cynomolgus monkeys were selected for this study based on their recently reported promising and comparable intrinsic clearance with human for BIBX1382 (Hutzler et al., 2014), which has been in agreement with several earlier studies (Kawashima et al., 1999; Itoh et al., 2006b; Diamond et al., 2010; Garattini and Terao, 2012).

Numerical fitting of kinetic profiles allows for flexibility in data analysis and an enhanced understanding of kinetic mechanisms. Kinetic modeling is the method of choice over the analytical Michaelis-Menten method to study enzymes with atypical kinetics such as AO. Traditional kinetic methods rely on several assumptions, such as the steady state hypothesis that limits and occasionally misdirects our understanding of the kinetic data. Based on our previous studies on the human aldehyde oxidase (hAO), we have found that this enzyme shows a nonlinear behavior and should be assessed numerically to take the whole kinetic process into account instead of truncating the data to meet the MichaelisMenten assumptions (Abbasi et al., 2019). Here, we use the same numerical approach to investigate the basis of interspecies kinetic differences between human and cynomolgus monkey.

\section{Materials and Methods}

\section{Chemicals and Reagents}

$\mathrm{O}^{6}$-benzylguanine (O6BG) and its metabolite, 8-oxo-benzylguanine, were purchased from Toronto Research Chemicals (North York, ON). Dantrolene was purchased from Cayman Chemical Company (Ann Arbor, MI), and the metabolite, aminodantrolene (ADNTN), was synthesized as described elsewhere (Amano et al., 2018). Phenacetin, glucose oxidase (Aspergillus spp.), glucose, and catalase (bovine liver) were purchased from Sigma-Aldrich (St. Louis, MO). The monobasic potassium phosphate, dibasic potassium phosphate, and EDTA used for the potassium phosphate buffer were purchased from JT Baker (Center Valley, PA).

\section{Site-Directed Mutagenesis and cDNA Analysis}

The QuikChange II site-directed mutagenesis kit from Agilent (Santa Clara, CA) was used to make the single and double mutants on the codon-optimized pTHco-hAOX1 plasmid (Foti et al., 2016). The primers (Table 1) for this study were designed through the Agilent's QuikChange primer design platform and bought from Invitrogen. The resulting polymerase chain reaction products from following the QuikChange mutagenesis protocol were purified using the Wizard plus midiprep DNA purification system. The sequence analysis was performed using a 3730 DNA Analyzer and the BigDye Terminator ready reaction mix from Applied Biosystems (Foster City, CA). A BLAST sequence alignment was performed to confirm the mutations. To make the FLVA double mutant, the purified F885L plasmid was used as the DNA template to be mutated once more with the primers designed to incorporate the V811A mutation.

\section{Expression of AO in Escherichia coli}

Expression and purification of hAO was performed according to the method previously described (Alfaro et al., 2009; Paragas et al., 2017b). The cynomolgus monkey aldehyde oxidase (mAO) plasmid was a gift from Professor Yorihisa Tanaka (Hoshino et al., 2007). The expression and purification process of $\mathrm{mAO}$ as well as of the three mutants was similar to that of hAO. The total amount of the purified enzyme was quantified by liquid chromatography-tandem mass spectrometry (Barr et al., 2013).

\section{Time Course Kinetic Assays}

All the Assays Were Done Under Anaerobic Conditions. Thirty units per milliliter final concentration of glucose oxidase and $150 \mathrm{U} / \mathrm{ml}$ of catalase were added to each reaction vial containing saturating amounts (five times the $\mathrm{K}_{\mathrm{m}}$ ) of O6BG $(200 \mu \mathrm{M})$ and dantrolene $(30 \mu \mathrm{M})$ dissolved in $0.1 \mathrm{M}$ potassium phosphate buffer ( $\mathrm{pH}$ 7.4) containing $0.1 \mathrm{mM}$ EDTA. Next, $50 \mathrm{mM}$ of glucose was added to each incubation after they were prewarmed for 5 minutes at $37^{\circ} \mathrm{C}$ to start the depletion of the molecular oxygen in the reaction. The biotransformation would then be initiated after 5 minutes by adding $1 \mu l$ of the purified enzyme. The final AO concentration for hAO, mAO, F885L, V811M, and FLVA was 38.4, $10.1,28.6,45.0$, and $46.3 \mathrm{nM}$, respectively. The total reaction volume was $1.5 \mathrm{ml}$ and $100 \mu l$ of it was quenched at $0.2,3,10,20,30,45,60,90$, and 120 minutes in $25 \mu \mathrm{l}$ of $1 \mathrm{M}$ formic acid containing $10 \mu \mathrm{M}$ phenacetin as an internal standard. The enzyme assays were all done in triplicates, and the goodness of fit was evaluated using the $R^{2}$ and Akaike information criterion (AIC) values.

\section{Liquid Chromatography-Mass Spectrometry}

Samples were analyzed using an LC-20AD series high-performance liquid chromatography system (Shimadzu, Columbia, MD) fitted with an HTC PAL autosampler (LEAP Technologies, Carrboro, NC). Chromatography was performed using a Kinetex $2.6 \mu \mathrm{m} \mathrm{C18} 100 \AA$ column $(100 \times 2.1 \mathrm{~mm}$; Phenomenex). Mobile phase A consisted of $0.05 \%$ (by volume) formic acid and $0.2 \%$ acetic acid (MilliporeSigma, Billerica, MA) in water, and mobile phase 
A

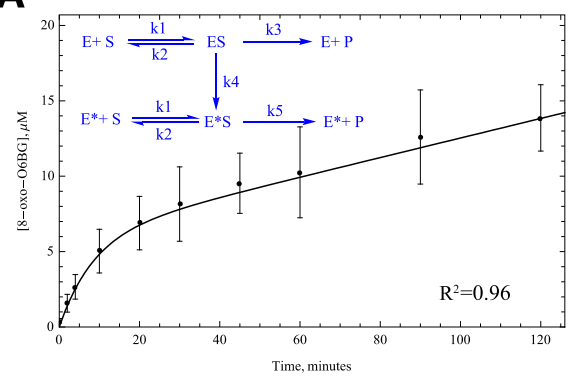

c

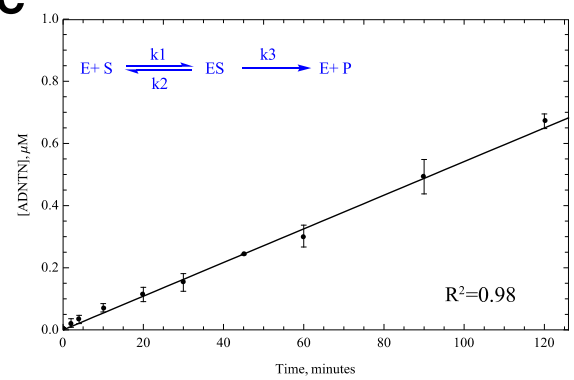

B

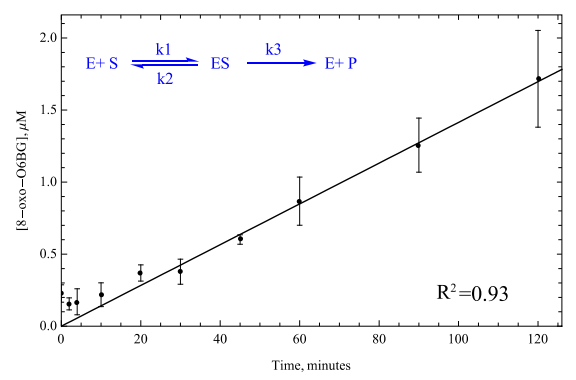

D

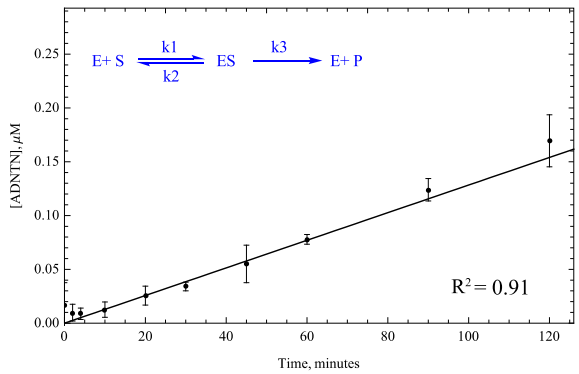

Fig. 1. The time course plot of the product formation under anaerobic conditions using glucose oxidase to remove oxygen (as described in Materials and Methods) is presented for O6BG in $\mathrm{hAO}(\mathrm{A})$, O6BG in mAO (B), dantrolene in $\mathrm{hAO}(\mathrm{C})$, and dantrolene in $\mathrm{mAO}(\mathrm{D})$. Saturating concentration (five times the $\mathrm{K}_{\mathrm{m}}$ ) of both oxidative (O6BG, $200 \mu \mathrm{M})$ and reductive (dantrolene, $30 \mu \mathrm{M}$ ) substrates were used for these kinetic assays. The kinetic models used in fitting these data sets are presented as blue insets, and the $R^{2}$ values are mentioned on the bottom right side of each graph. The MAM was used for (A), and the Michaelis-Menten linear model was used for (B-D). Models were selected according to the kinetic trend of the product formation in each data set.
B comprised $90 \%$ acetonitrile (MilliporeSigma), 9.9\% water, and $0.1 \%$ formic acid (Fischer Scientific, Pittsburgh, PA). The quantitation was conducted on an API 4000 Q-Trap mass spectrometry system (Applied Biosystems/MDS Sciex) with turbo spray electrospray ionization operating in positive ion mode. The chromatography for all the analytes started at $10 \%$ mobile B concentration and would ramp up to $75 \%$ mobile $\mathrm{B}$ in 6 minutes, and the concentration was maintained for half a minute before ramping back down to $10 \%$ mobile $\mathrm{B}$ in 1 minute. The mass spectrometry tuning parameters used were as follows: curtain gas, 20; ion spray voltage, 4900 ; desolvation temperature, $600^{\circ} \mathrm{C}$; ion source gas 1, 35; ion source gas 2, 55; collision gas, medium; declustering potential, 70; entrance potential, 10; collision energy, 35; cell exit potential, 15. The flow rate was $0.25 \mathrm{ml} / \mathrm{min}$, and the metabolites and internal standard were detected using multiple reaction monitoring mode following these $\mathrm{m} / \mathrm{z}$ transitions: 8-oxobenzylguanine, $258.2 \rightarrow 91.0 ;$ ADNTN, 285.1 $\rightarrow 186.0$ and phenacetin (internal standard), $180.2 \rightarrow 110.1$.

\section{Data Analysis}

Numerical fitting in Mathematica 11.0.1.0 (Wolfram Research, Champaign, IL) was performed using the NDSolve function with MaxSteps $\rightarrow 10,000$ and PrecisionGoal $\rightarrow \infty ; \mathrm{k}_{1}$ was fixed to $270 \mu \mathrm{M} \mathrm{s}^{-1}$, and $\mathrm{k}_{2}$ was set to $\mathrm{k}_{1} \times \mathrm{K}_{\mathrm{m}}$ for each substrate. The $\mathrm{K}_{\mathrm{m}}$ for each substrate was derived from the data on file and was set to 70 for O6BG in hAO, 7 in mAO, and $10 \mu \mathrm{M}$ for the mutants. For dantrolene, the $K_{m}$ was set to $5 \mu \mathrm{M}$ for all the enzyme sources. Model fitting was done using the NonlinearModelFit function with $1 / Y$ weighting. The goodness of fit was evaluated by AIC and $R^{2}$ commands. The models used were the modified activity model (MAM) and the linear (Michaelis-Menten) model as described previously (Abbasi et al., 2019). The decision on what model to choose for each data set was made by comparing the AIC obtained through the fits to each model.

\section{Results}

Kinetic Modeling. Here, we used a kinetic modeling approach to determine the rate of oxidation and reduction half-reactions for AO. For the oxidation half-reaction, we used O6BG as a substrate. Oxygen is a challenging substrate to probe. Therefore, to remove any complexities raised by competition between the reductive substrates, we substituted oxygen with dantrolene as a reducing substrate of AO under anaerobic conditions to probe the reduction half-reaction in this enzyme (Amano et al., 2018) as described in the Materials and Methods section. We chose dantrolene as the reducing substrate in this study based on the previous reports of this compound being metabolized by AO even under normal oxygen concentration as well as its lack of competition with oxidative substrates and having a high turnover rate among other structurally similar reductive substrates of AO (Amano et al., 2018; Ogiso et al., 2018). Furthermore, dantrolene has a similar $\mathrm{K}_{\mathrm{m}}$ to oxygen, with the $\mathrm{K}_{\mathrm{m}}$ of oxygen for hAO being estimated to be close to $2 \mu \mathrm{M}$ (Abbasi et al., 2019) and the $\mathrm{K}_{\mathrm{m}}$ of dantrolene being $5 \mu \mathrm{M}$ (data not shown).

TABLE 2

A comparison between the rate of product formation between the two species as well as the mutants under anaerobic conditions using a glucose oxidase system as described in the Materials and Methods section.

MAM was the model of choice in fitting the O6BG $(200 \mu \mathrm{M})$ product formation for hAO and all the mutants and Michaelis-Menten linear model was used to fit the O6BG product formation in $\mathrm{mAO}$ as well as the product formation for dantrolene $(30 \mu \mathrm{M})$ in both species and the mutants.

\begin{tabular}{|c|c|c|c|c|}
\hline & \multicolumn{3}{|c|}{ Rate of 8-oxo-O6BG formation } & \multirow{2}{*}{$\begin{array}{l}\text { Rate of ADNTN formation } \\
\begin{array}{c}k_{3 \_ \text {red }} \\
\text { min }^{-1}\end{array}\end{array}$} \\
\hline & $\begin{array}{l}\mathrm{k}_{3 \text {-ox }} \\
\min ^{-1}\end{array}$ & $\underset{\min ^{-1}}{\mathrm{k}_{4}}$ & $\begin{array}{c}\mathrm{k}_{5} \\
\min ^{-1}\end{array}$ & \\
\hline hAO & $110 \pm 20.7$ & $0.160 \pm 0.0494$ & $9.28 \pm 2.03$ & $0.659 \pm 0.0182$ \\
\hline $\mathrm{mAO}$ & $2.19 \pm 0.0696$ & - & - & $0.156 \pm 0.00876$ \\
\hline F885L & $38.7 \pm 3.85$ & $0.0388 \pm 0.0144$ & $7.90 \pm 2.71$ & $0.701 \pm 0.0193$ \\
\hline V811A & $40.7 \pm 8.51$ & $0.158 \pm 0.0486$ & $2.95 \pm 0.522$ & $0.336 \pm 0.0143$ \\
\hline FLVA & $30.4 \pm 5.02$ & $0.0659 \pm 0.0289$ & $7.50 \pm 1.37$ & $0.486 \pm 0.0199$ \\
\hline
\end{tabular}


A

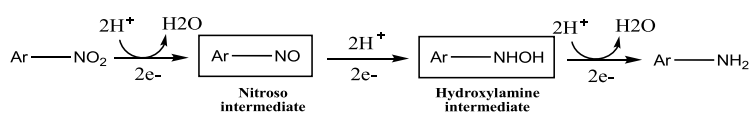

B

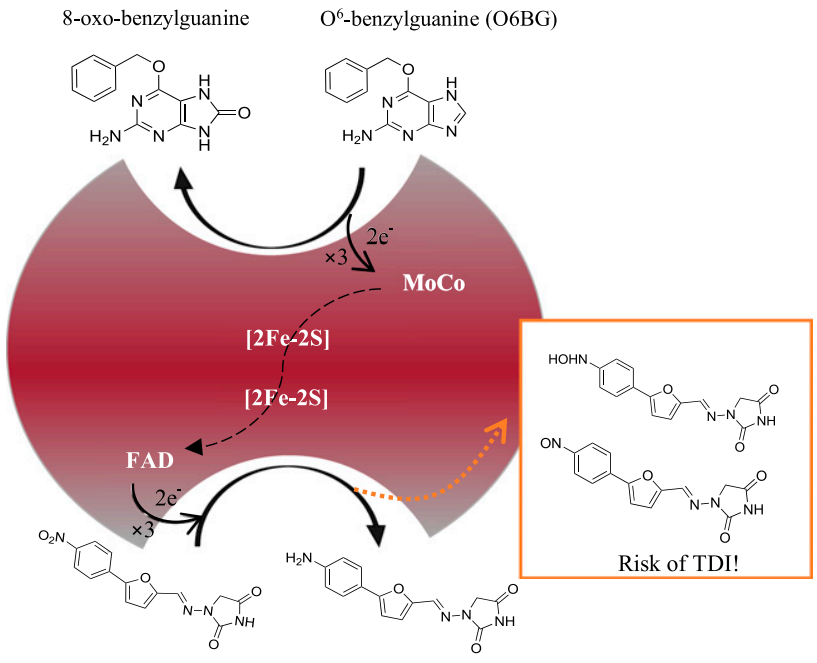

dantrolene

aminodantrolene (ADNTN)

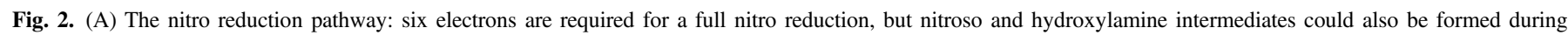

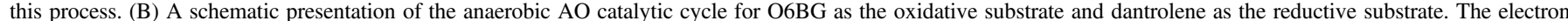

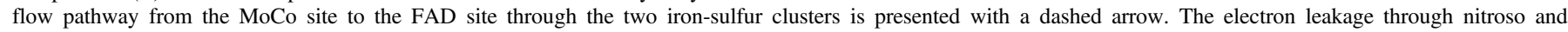

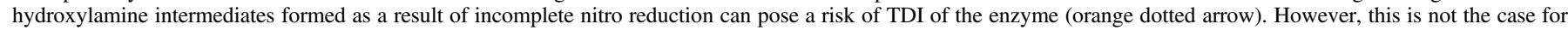
O6BG oxidation by AO.

Analysis of 8-oxo-O6BG formation over time demonstrates that whereas hAO has biphasic kinetics, $\mathrm{mAO}$ is linear (Fig. 1, A and B). The MAM model was used to account for the curvature in the rate of 8-oxoO6BG formation in hAO. Numerical fitting to MAM solves for the unknown rate constants $\mathrm{k}_{3 \_ \text {ox }}$ and $\mathrm{k}_{5}$ in the model with $\mathrm{k}_{3 \_ \text {ox }}$ representing the initial fast oxidation rate in $\mathrm{hAO}$ and $\mathrm{k}_{5}$ representing the turnover rate during the second phase of catalysis where reduction is assumed to be the rate-limiting step (Fig. 1A, kinetic scheme inset). It is important to note that $\mathrm{k}_{5}$ is used to represent the rate of reduction and/or the rate of enzyme reoxidation interchangeably throughout this paper. This biphasic kinetic behavior does not exist for O6BG metabolism in mAO (Fig. 1B); neither does it exist for the dantrolene progress curves in either of the species (Fig. 1, C and D). Therefore, we used a linear MichaelisMenten model to numerically fit the time course data in those plots. In the Michaelis-Menten model, $\mathrm{k}_{3}$ represents the rate of product formation ( $k_{3 \text { ox }}$ for the 8-oxo-O6BG formation and $k_{3 \text { red }}$ for ADNTN formation). Both of the substrates, O6BG and dantrolene, were present at their saturating level (five times the $\mathrm{K}_{\mathrm{m}}$ or more) in the reaction to remove the possibility of substrate depletion. Therefore, the rates presented in Table 2 are representative of the maximum rate of reaction. Based on the comparison between the rate of product formation during each of the catalytic half-reactions under anaerobic conditions, there is almost a 166-fold difference between the oxidation rate $\left(\mathrm{k}_{3}\right.$ ox $)$ from fitting the O6BG oxidation to MAM and the reduction rate $\left(\mathrm{k}_{3}\right.$ _red $)$ derived from fitting the dantrolene reduction to the Michaelis-Menten model in hAO. On the other hand, the $\mathrm{k}_{3 \text { ox }}$ is only 14-fold higher than the $\mathrm{k}_{3 \text { red }}$ in mAO. Also, despite the rate of oxidation $\left(\mathrm{k}_{3}\right.$ ox $)$ in human being 50 -fold faster than monkey, there is a much lower 4-fold difference in the rate of reduction $\left(\mathrm{k}_{3 \_ \text {red }}\right)$ between the two species.

Probing the Efficacy of Reduction in AO Under Anaerobic Conditions. To compare the efficacy of reduction between human and monkey, we have calculated the ratio of the concentration of the oxidized product (8-oxo-O6BG) to the concentration of the reduced product (ADNTN) formed during the enzymatic reaction and called this ratio OAR. AO performs nitro reduction on dantrolene and for a nitro group to be reduced, six electrons are required (Fig. 2A). In a perfect and $100 \%$ efficient case, the OAR would be equal to three since each round of O6BG oxidation provides two electrons out of the total of six electrons required to form ADNTN. The greater the electron leakage in the catalytic cycle is, the higher this ratio goes. An example of electron leakage could be a case of incomplete nitro reduction leading to the release of hydroxylamine and nitroso-containing intermediates. A schematic presentation of the AO catalytic cycle and the electron flow pathway is demonstrated in Fig. 2B. Under anaerobic conditions and five times the $\mathrm{K}_{\mathrm{m}}$ amount of O6BG and dantrolene, the OAR is close to an average of $50.3 \pm 20.7$ in hAO, whereas it holds a much closer to the ideal value of $3(5.8 \pm 1.8)$ for mAO (Table 3$)$. This means that the efficacy of electron shuttle and reduction is almost 10 -fold higher in mAO than hAO. It is also important to note that the efficacy of the electron shuttle or the balance between the enzymatic oxidation and reduction is the result of the combination of the binding affinity of the substrates as well as their rate of enzymatic turnover. We have performed all of the experiments using a saturating amount of each substrate to only compare the maximum rate of reaction in all cases.

Molecular Basis of Interspecies Kinetic Differences. To establish whether certain amino acids at the MoCo active site were responsible for the kinetic differences between hAO and mAO, we performed sitedirected mutagenesis on hAO to make it more like mAO. Having as much as $95.1 \%$ sequence identity (Hoshino et al., 2007) between hAO and $\mathrm{mAO}$ limits the number of amino acid differences to be considered to 58. We inspected the hAO crystal structure (Protein Data Bank identifier $4 \mathrm{UHW}$ ) trying to pinpoint amino acid differences between human and cynomolgus monkey (hAO\#mAO) close to the cofactors. We found that six of the differences are in the vicinity of the MoCo site: K661E, V811A, L812F, I816V, F885L, and F1014Y. One further mutation, E274Q, is at the FAD domain at $8 \AA$ from one of the FAD phosphates, being an exposed residue. We singled out the V811A and F885L out of only six MoCo-adjacent mutants as well as their double mutant (FLVA) for this investigation since the phenylalanine and valine residues had been reported to play an important role in orienting the substrate at the MoCo site (Coelho et al., 2015; Terao et al., 2020).

Similar to what was observed for hAO and mAO, the dantrolene reduction progress curves under anaerobic conditions and with saturating amounts of O6BG and dantrolene remained linear with time for the 


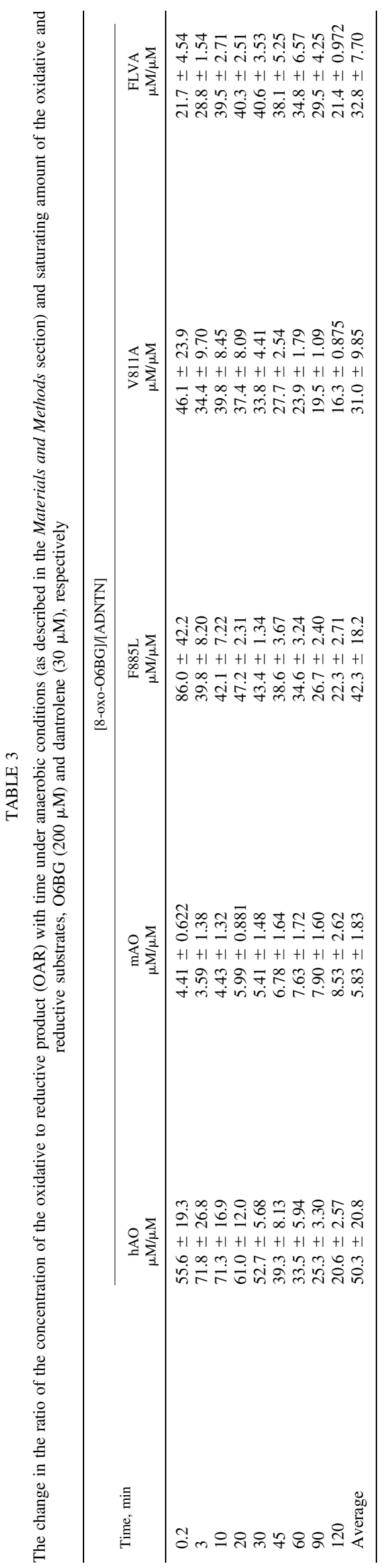

mutants as well (Fig. 3). The initial visual assessment of the mutants' O6BG oxidation progress curves from the same reaction (Fig. 4) suggested a small shift toward a more linear monkey-like kinetic behavior. However, nonlinear kinetics were still observed for all the mutants, and based on the AIC, the MAM gave a better fit to both F885L and V811A single mutants as well as their double mutant (FLVA) in comparison with the linear model (Fig. 4). We used the OAR criteria to assign human-like or monkey-like characteristics to all the mutated hAOs more quantitatively. At both ends of the spectrum stands hAO with an average OAR value of $50.3 \pm 20.8$ and $\mathrm{mAO}$ with an average OAR value of $5.83 \pm 1.83$. Although all of the F885L, V811A, and FLVA mutants hold a lower average OAR value than hAO, $42.3 \pm 18.2$, $31.0 \pm 9.85$, and $32.8 \pm 7.70$, respectively, their OAR valueS was still much closer to human than that of the cynomolgus monkey.

\section{Discussion}

AO has proven to be a challenging drug metabolizing enzyme for clearance estimation in humans. Not only is AO both substrate and species specific, it also exhibits nonlinear atypical kinetics (Dalvie et al., 2010; Barr and Jones, 2013; Choughule et al., 2015; Abbasi et al., 2019). With more than $95.1 \%$ sequence identity between human and cynomolgus monkey AO, cynomolgus monkey may be the best species to estimate AO clearance in human. The comparable AO-mediated metabolism of BIBX1382, SGX523, VU0409106, RS-8359, and zaleplon between human and cynomolgus monkey is also consistent with this notion (Kawashima et al., 1999; Itoh et al., 2006b; Diamond et al., 2010; Garattini and Terao, 2012; Morrison et al., 2012; Hutzler et al., 2013, 2014).

Our previous studies on numerous substrates of hAO have shown that this enzyme exhibits nonlinear, biphasic kinetics (Abbasi et al., 2019). We have assigned the fast initial phase to the rate of substrate oxidation and the second slower phase to the stage during the enzymatic reaction where the enzyme reoxidation is the rate-limiting step. Because the linear Michaelis-Menten model performs poorly with nonlinear data, we have modeled this biphasic kinetics using MAM (blue inset in Fig. 1A). In this kinetic scheme, the fast initial phase is presented by the $\mathrm{k}_{3}$ value, and the slower phase is captured by the $\mathrm{k}_{5}$ micro rate constant. What this means is that once all the hAO enzyme molecules in the reaction are reduced by the oxidizing substrates (e.g., O6BG), the reaction mostly proceeds at the rate of enzymatic reoxidation $\left(\mathrm{k}_{5}\right)$ rather than the rate of substrate oxidation $\left(\mathrm{k}_{3 \_ \text {ox }}\right)$. In contrast, linear kinetics was observed with $\mathrm{mAO}$.

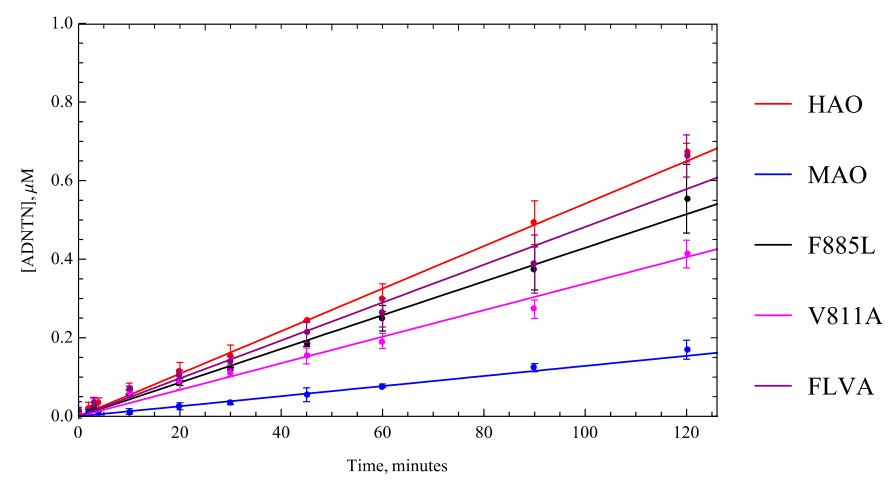

Fig. 3. The time course plot of the ADNTN formation under anaerobic conditions using a saturating amount of O6BG $(200 \mu \mathrm{M})$ as the oxidative substrate and dantrolene as the reductive substrate $(30 \mu \mathrm{M})$. A glucose oxidase system was used to remove oxygen (as described in Materials and Methods) in all of the anaerobic reactions. The reductive half-reaction remained linear and was fit to the MichaelisMenten model in all the mutants, F885L (black, $R^{2}=0.98$ ), V811A (magenta, $R^{2}=$ 0.95 ), and FLVA (purple, $R^{2}=0.96$ ), similar to hAO (red, $R^{2}=0.98$ ) and mAO (blue, $\left.R^{2}=0.91\right)$. 
A

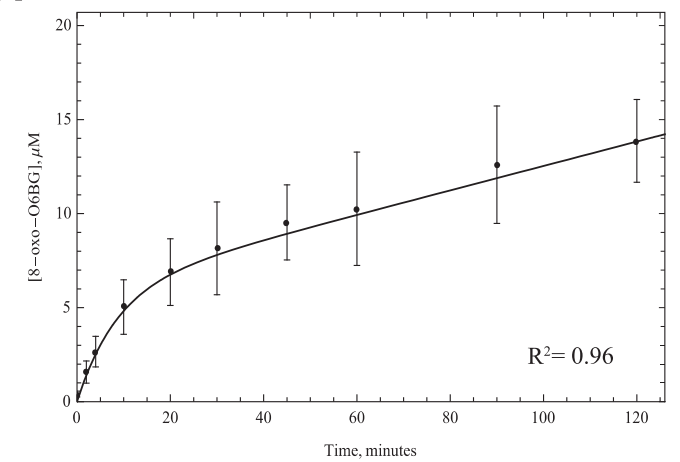

C

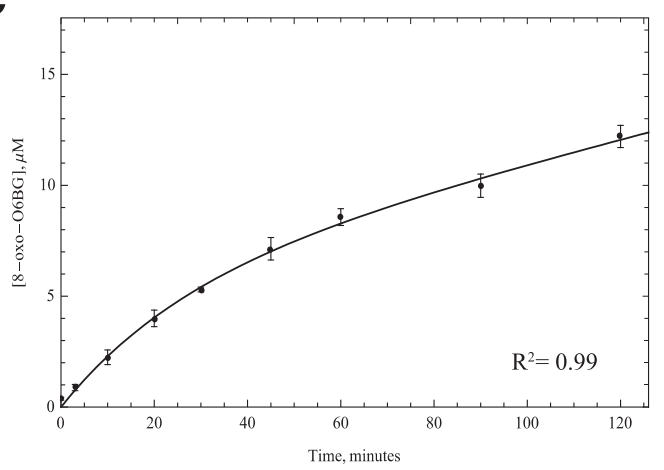

B

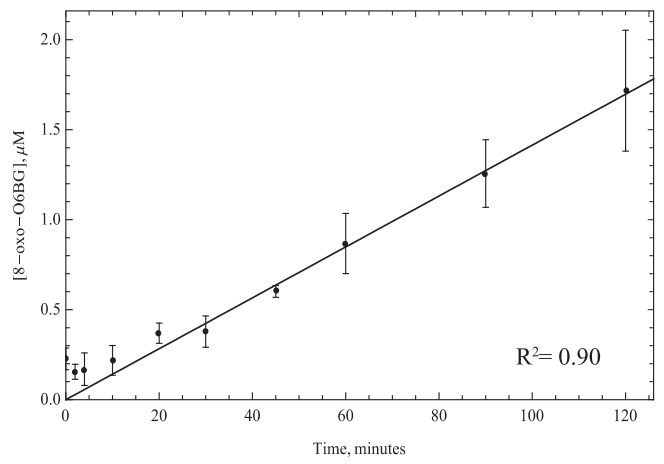

D

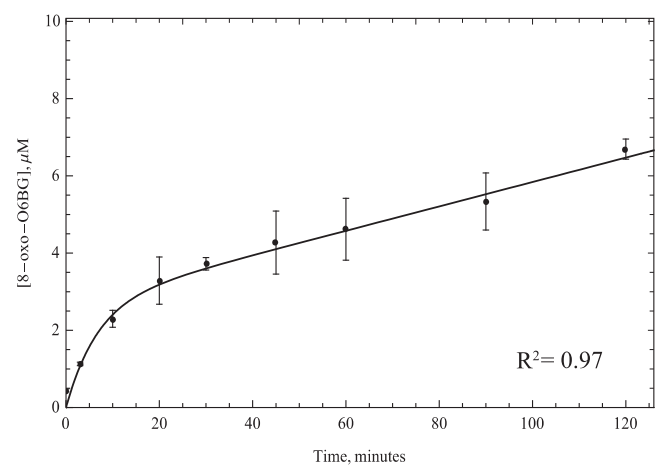

$\mathbf{E}$

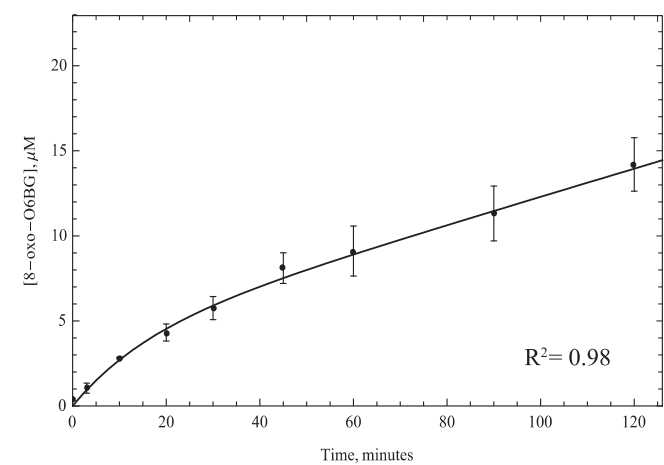

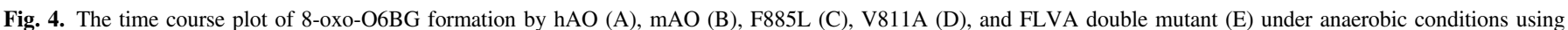

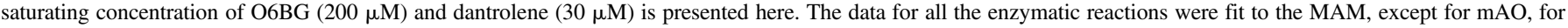
which the linear Michaelis-Menten model was used.

If the traditional method of determining the linear region of the progress curves is applied to derive the rate of the reaction, a significant amount of information that mechanistically differentiates hAO from $\mathrm{mAO}$ is lost. Herein we take the full time course into account to describe the differences in the AO metabolic progress curves for O6BG between monkey (linear) and human $\mathrm{AO}$ (nonlinear). This makes the comparison and identification of the molecular basis of their differences more feasible since rates are difficult to directly compare. Therefore, any structural differences between $\mathrm{hAO}, \mathrm{mAO}$, and their mutants mirrored in the progress curves would be visually easy to identify. Since there is a much smaller almost 14 -fold difference between $\mathrm{k}_{3 \_o x}$ and $\mathrm{k}_{3 \text { _red }}$ in $\mathrm{mAO}$ as opposed to a 166 -fold difference in hAO, the linearity of the $\mathrm{mAO}$ progress curve can be attributed to a balance between the rate of the two catalytic half-reactions (Table 2). With the reduction as the ratelimiting step, there is a lower chance of electron backlog in mAO with a much slower oxidation rate in comparison with hAO. The same results were obtained with oxygen as the electron acceptor (Supplemental
Table 1), eliminating the possibility of this kinetic discrepancy simply being the result of using a nonendogenous electron acceptor or having reductive intermediates.

Since both hAO and $\mathrm{mAO}$ have shown similar linear reductive kinetic behavior, we have decided to look into whether the interspecies kinetic differences can be explained by the differences in the oxidation binding site. The MoCo site of the human AO has been identified as the oxidative substrate's binding region. Therefore, we have first looked into the amino acid differences between the two species around this site. The two amino acids chosen for this study were the F885 and V811 residues, which were identified as important amino acids in orienting the substrate toward the catalytic site in human $\mathrm{AO}$ and stabilization of the intermediate MoCo species (Coelho et al., 2015; Terao et al., 2020).

The reduction is believed to be the rate-limiting step in AO catalytic cycle (Abbasi et al., 2019). Therefore, a comparison between the $\mathrm{k}_{3 \_ \text {red }}$ values in hAO and $\mathrm{mAO}$ as well as their mutants is of high importance (Table 2). Although this value in F885L mutant remains the same as 
hAO, the V811A-containing mutants seem to shift toward a more monkey-like, lower rate of reduction similar to $\mathrm{mAO}$. However, since there is a catalytic crosstalk between the reduction and oxidation halfreactions, the nonlinearity of the progress curves in mutants is the result of an imbalance between $\mathrm{k}_{3 \_\mathrm{ox}}$ and $\mathrm{k}_{3 \_ \text {red }}$ similar to what was observed in $\mathrm{hAO}$, and this nonlinearity is not solely dependent to $\mathrm{k}_{3 \_ \text {red }}$.

In an attempt to quantitatively assess the differences between hAO, $\mathrm{mAO}$, and their mutants, we have defined the OAR criteria as the stoichiometric ratio of [8-oxo-O6BG]/[ADNTN] made during the enzymatic reaction in the absence of oxygen. As shown in Fig. 2, three oxidation reactions are required to fully reduce the nitro group in dantrolene to an amine. Experiments were done anaerobically to decrease the complexity of this analysis by removing an alternative electron acceptor (molecular oxygen) from the system. Therefore, a potential remaining pathway of electron leakage could be the formation of the reductive intermediates of dantrolene such as hydroxylamine and nitroso species. These intermediates could leave the reductive site before a complete nitro reduction into ADNTN occurs. Although these compounds are reactive intermediates posing a risk of time-dependent inhibition (TDI) (Yadav et al., 2020), there is no correlation between the biphasic kinetics for O6BG in hAO and TDI since such nonlinearity does not exist for the reductive half-reaction for dantrolene in this enzyme (Fig. 3). In this sense, OAR could be used as the means to gauge the efficiency of electron shuttle from the oxidative site to the reductive site of the enzyme. The route that the electrons have to take is believed to be from the MoCo site to the FAD site and through the two iron-sulfur clusters that act as electron sinks during catalysis (Pryde et al., 2010). Based on our results, OAR holds a much smaller value in cynomolgus monkey, followed by V811A, FLVA, F885L, and human AO (Table 3). Overall, although we saw some progress toward a more monkey-like kinetics in the mutants we explored (especially for the V811A-containing mutants), other amino acids must also play a role in the kinetic differences between human and cynomolgus monkey AO.

Several other potentially important amino acid differences, especially the K661E at the gate 1 of the substrate access channel, as well as L812F, which is another important amino acid difference pointing to the entrance of the active site pocket, still remain to be considered at the MoCo site of AO (Coelho et al., 2015). However, recent publications regarding the $\mathrm{AO}$ nitro reduction, most notably the one by Paragas et al. in which the FAD site has been suggested as the reductive site of this enzyme, have brought up the possibility of amino acids at this site also having an important role in causing this marked kinetic difference between the two species (Konishi et al., 2017; Paragas et al., 2017a; Amano et al., 2018; Ogiso et al., 2018). However, it is also important to note that there are two sites involved in playing this catalytic seesaw. Therefore, there is always the possibility of requiring a change in a combination of amino acids at both of the FAD and MoCo sites in hAO to arrive at a kinetic behavior that is more similar to $\mathrm{mAO}$.

In conclusion, although hAO and mAO share a high degree of sequence similarity, they do not share common kinetics, and mAO may not be a good model for hAO. Attempts to understand why hAO and $\mathrm{mAO}$ are different, using site-directed mutagenesis of amino acids in the MoCo active site, showed some progress toward slower monkey-like kinetics especially in the V811A-containing mutants. However, we believe that differences in the structure outside the MoCo active site may also be responsible for the differences in kinetics.

\section{Acknowledgments}

We thank Dr. Sara Humphreys for her help in proofreading this manuscript and her insightful comments.

\section{Authorship Contributions}

Participation in research design: Abbasi, Jones.

Conducted experiments: Abbasi, Joswig-Jones.

Performed data analysis: Abbasi, Jones.

Wrote or contributed to the writing of the manuscript: Abbasi, Jones.

\section{References}

Abbasi A, Paragas EM, Joswig-Jones CA, Rodgers JT, and Jones JP (2019) Time course of aldehyde oxidase and why it is nonlinear. Drug Metab Dispos 47:473-483.

Alfaro JF and Jones JP (2008) Studies on the mechanism of aldehyde oxidase and xanthine oxidase. J Org Chem 73:9469-9472.

Alfaro JF, Joswig-Jones CA, Ouyang W, Nichols J, Crouch GJ, and Jones JP (2009) Purification and mechanism of human aldehyde oxidase expressed in Escherichia coli. Drug Metab Dispos 37:2393-2398.

Amano T, Fukami T, Ogiso T, Hirose D, Jones JP, Taniguchi T, and Nakajima M (2018) Identification of enzymes responsible for dantrolene metabolism in the human liver: a clue to uncover the cause of liver injury. Biochem Pharmacol 151:69-78.

Barr JT and Jones JP (2013) Evidence for substrate-dependent inhibition profiles for human liver aldehyde oxidase. Drug Metab Dispos 41:24-29.

Barr JT, Jones JP, Joswig-Jones CA, and Rock DA (2013) Absolute quantification of aldehyde oxidase protein in human liver using liquid chromatography-tandem mass spectrometry. Mol Pharm 10:3842-3849.

Cheshmazar N, Dastmalchi S, Terao M, Garattini E, and Hamzeh-Mivehroud M (2019) Aldehyde oxidase at the crossroad of metabolism and preclinical screening. Drug Metab Rev 51:428-452 DOI: $10.1080 / 03602532.2019 .1667379$.

Choughule KV, Barr JT, and Jones JP (2013) Evaluation of rhesus monkey and guinea pig hepatic cytosol fractions as models for human aldehyde oxidase. Drug Metab Dispos 41:1852-1858.

Choughule KV, Joswig-Jones CA, and Jones JP (2015) Interspecies differences in the metabolism of methotrexate: an insight into the active site differences between human and rabbit aldehyde oxidase. Biochem Pharmacol 96:288-295.

Coelho C, Foti A, Hartmann T, Santos-Silva T, Leimkühler S, and Romão MJ (2015) Structural insights into xenobiotic and inhibitor binding to human aldehyde oxidase. Nat Chem Biol 11: 779-783.

Crouch RD, Hutzler JM, and Daniels JS (2018) A novel in vitro allometric scaling methodology for aldehyde oxidase substrates to enable selection of appropriate species for traditional allometry. Xenobiotica 48:219-231.

Dalvie D, Xiang C, Kang P, and Zhou S (2013) Interspecies variation in the metabolism of zoniporide by aldehyde oxidase. Xenobiotica 43:399-408

Dalvie D, Zhang C, Chen W, Smolarek T, Obach RS, and Loi CM (2010) Cross-species comparison of the metabolism and excretion of zoniporide: contribution of aldehyde oxidase to interspecies differences. Drug Metab Dispos 38:641-654

Davydova NY, Dangi B, Maldonado MA, Vavilov NE, Zgoda VG, and Davydov DR (2019) Toward a systems approach to cytochrome P450 ensemble: interactions of CYP2E1 with other P450 species and their impact on CYP1A2. Biochem J 476:3661-3685.

Diamond S, Boer J, Maduskuie TP Jr, Falahatpisheh N, Li Y, and Yeleswaram S (2010) Speciesspecific metabolism of SGX523 by aldehyde oxidase and the toxicological implications. Drug Metab Dispos 38:1277-1285.

Dittrich Ch, Greim G, Borner M, Weigang-Köhler K, Huisman H, Amelsberg A, Ehret A, Wanders J, Hanauske A, and Fumoleau P (2002) Phase I and pharmacokinetic study of BIBX 1382 BS, an epidermal growth factor receptor (EGFR) inhibitor, given in a continuous daily oral administration. Eur J Cancer 38:1072-1080.

Dowers TS, Rock DA, Rock DA, Perkins BNS, and Jones JP (2004) An analysis of the regioselectivity of aromatic hydroxylation and N-oxygenation by cytochrome P450 enzymes. Drug Metab Dispos 32:328-332.

Foti A, Hartmann T, Coelho C, Santos-Silva T, Romão MJ, and Leimkühler S (2016) Optimization of the expression of human aldehyde oxidase for investigations of single-nucleotide polymorphisms. Drug Metab Dispos 44:1277-1285.

Fukiya K, Itoh K, Yamaguchi S, Kishiba A, Adachi M, Watanabe N, and Tanaka Y (2010) A single amino acid substitution confers high cinchonidine oxidation activity comparable with that of rabbit to monkey aldehyde oxidase 1. Drug Metab Dispos 38:302-307.

Garattini E, Fratelli M, and Terao M (2009) The mammalian aldehyde oxidase gene family. Hum Genomics 4:119-130.

Garattini E and Terao M (2012) The role of aldehyde oxidase in drug metabolism. Expert Opin Drug Metab Toxicol 8:487-503.

Hoshino K, Itoh K, Masubuchi A, Adachi M, Asakawa T, Watanabe N, Kosaka T, and Tanaka Y (2007) Cloning, expression, and characterization of male cynomolgus monkey liver aldehyde oxidase. Biol Pharm Bull 30:1191-1198.

Hutzler JM, Cerny MA, Yang YS, Asher C, Wong D, Frederick K, and Gilpin K (2014) Cynomolgus monkey as a surrogate for human aldehyde oxidase metabolism of the EGFR inhibitor BIBX1382. Drug Metab Dispos 42:1751-1760.

Hutzler JM, Obach RS, Dalvie D, and Zientek MA (2013) Strategies for a comprehensive understanding of metabolism by aldehyde oxidase. Expert Opin Drug Metab Toxicol 9: $153-168$

Itoh K, Hoshino K, Endo A, Asakawa T, Yamakami K, Noji C, Kosaka T, and Tanaka Y (2006a) Chiral inversion of RS-8359: a selective and reversible MAO-A inhibitor via oxido-reduction of keto-alcohol. Chirality 18:698-706.

Itoh K, Yamamura M, Takasaki W, Sasaki T, Masubuchi A, and Tanaka Y (2006b) Species differences in enantioselective 2-oxidations of RS-8359, a selective and reversible MAO-A inhibitor, and cinchona alkaloids by aldehyde oxidase. Biopharm Drug Dispos 27:133-139.

Kawashima K, Hosoi K, Naruke T, Shiba T, Kitamura M, and Watabe T (1999) Aldehyde oxidasedependent marked species difference in hepatic metabolism of the sedative-hypnotic, zaleplon, between monkeys and rats. Drug Metab Dispos 27:422-428.

Konishi K, Fukami T, Gotoh S, and Nakajima M (2017) Identification of enzymes responsible for nitrazepam metabolism and toxicity in human. Biochem Pharmacol 140:150-160.

Li H, Kundu TK, and Zweier JL (2009) Characterization of the magnitude and mechanism of aldehyde oxidase-mediated nitric oxide production from nitrite. J Biol Chem 284:33850-33858 DOI: $10.1074 /$ jbc.M109.019125. 
Maia LB, Pereira V, Mira L, and Moura JJG (2015) Nitrite reductase activity of rat and human xanthine oxidase, xanthine dehydrogenase, and aldehyde oxidase: evaluation of their contribution to NO formation in vivo. Biochemistry 54:685-710 DOI: 10.1021/bi500987w.

Mendel RR (2009) Cell biology of molybdenum. Biofactors 35:429-434.

Morrison RD, Blobaum AL, Byers FW, Santomango TS, Bridges TM, Stec D, Brewer KA, Sanchez-Ponce R, Corlew MM, Rush R, et al. (2012) The role of aldehyde oxidase and xanthine oxidase in the biotransformation of a novel negative allosteric modulator of metabotropic glutamate receptor subtype 5. Drug Metab Dispos 40:1834-1845.

Mota C, Esmaeeli M, Coelho C, Santos-Silva T, Wolff M, Foti A, Leimkühler S, and Romão MJ (2019) Human aldehyde oxidase (hAOX1): structure determination of the Moco-free form of the natural variant G1269R and biophysical studies of single nucleotide polymorphisms. FEBS Open Bio 9:925-934.

Nair AB and Jacob S (2016) A simple practice guide for dose conversion between animals and human. J Basic Clin Pharm 7:27-31.

Ogiso T, Fukami T, Mishiro K, Konishi K, Jones JP, and Nakajima M (2018) Substrate selectivity of human aldehyde oxidase 1 in reduction of nitroaromatic drugs. Arch Biochem Biophys $\mathbf{6 5 9}$ $85-92$.

Paragas EM, Humphreys SC, Min J, Joswig-Jones CA, and Jones JP (2017a) The two faces of aldehyde oxidase: oxidative and reductive transformations of 5-nitroquinoline. Biochem Pharmacol 145:210-217.

Paragas EM, Humphreys SC, Min J, Joswig-Jones CA, Leimkühler S, and Jones JP (2017b) ecoAO: a simple system for the study of human aldehyde oxidases role in drug metabolism. ACS Omega 2:4820-4827.
Pryde DC, Dalvie D, Hu Q, Jones P, Obach RS, and Tran T-D (2010) Aldehyde oxidase: an enzyme of emerging importance in drug discovery. J Med Chem 53:8441-8460.

Sharma V and McNeill JH (2009) To scale or not to scale: the principles of dose extrapolation. $B r$ $J$ Pharmacol 157:907-921.

Terao M, Garattini E, Romão MJ, and Leimkühler S (2020) Evolution, expression, and substrate specificities of aldehyde oxidase enzymes in eukaryotes. J Biol Chem 17:5377-5389.

Weidert ER, Schoenborn SO, Cantu-Medellin N, Choughule KV, Jones JP, and Kelley EE (2014) Inhibition of xanthine oxidase by the aldehyde oxidase inhibitor raloxifene: implications for identifying molybdopterin nitrite reductases. Nitric Oxide 15:41-45 DOI: 10.1016/ j.freeradbiomed.2013.10.638

Yadav J, Paragas E, Korzekwa K, and Nagar S (2020) Time-dependent enzyme inactivation: numerical analyses of in vitro data and prediction of drug-drug interactions. Pharmacol Ther 206: 107449

Zhang X, Liu HH, Weller P, Zheng M, Tao W, Wang J, Liao G, Monshouwer M, and Peltz G (2011) In silico and in vitro pharmacogenetics: aldehyde oxidase rapidly metabolizes a p38 kinase inhibitor. Pharmacogenomics $J$ 11:15-24.

Zientek M, Jiang Y, Youdim K, and Obach RS (2010) In vitro-in vivo correlation for intrinsic clearance for drugs metabolized by human aldehyde oxidase. Drug Metab Dispos 38:1322-1327.

Address correspondence to: Dr. Jeffrey P. Jones, Department of Chemistry, Washington State University, Pullman, WA 99164-4630. E-mail: jpj@wsu.edu 